\title{
Trans-Zeatin inhibits UVB-induced matrix metalloproteinase-1 expression via MAP kinase signaling in human skin fibroblasts
}

\author{
BO YANG $^{1 *}, \mathrm{CHAO} \mathrm{JI}^{1 *}, \mathrm{JIAN} \mathrm{KANG}^{1}, \mathrm{WENQI} \mathrm{CHEN}^{1}, \mathrm{ZHIGANG} \mathrm{BI}^{1}$ and YINSHENG WAN ${ }^{2}$ \\ ${ }^{1}$ Jiangsu Province Key Laboratory of Molecular Immunopathology, the First Affiliated Hospital \\ of Nanjing Medical University, Nanjing 210029, Jiangsu Province, P.R. China; \\ ${ }^{2}$ Department of Biology, Providence College, Providence, RI 02918, USA
}

Received November 27, 2008; Accepted January 26, 2009

DOI: 10.3892/ijmm_00000164

\begin{abstract}
Ultraviolet (UV) irradiation induces the expression of matrix metalloproteinases (MMPs), disturbing the metabolism of extracellular matrix (ECM), and causes the characteristic changes of photoaging in skin. Inhibition of induction of MMPs is suggested to alleviate photoaging induced by UV irradiation. Zeatin, purified from Zea mays, is a member of the cytokinin group of plant growth factors, the activity of which is attributed to its more stable trans form. In this study, we investigated the effect of trans-Zeatin on UVBinduced matrix metalloproteinase-1 (MMP-1) expression in cultured human skin fibroblasts (HSFs) and studied the mechanisms of its actions. We found that pretreatment with trans-Zeatin significantly inhibits UVB-induced MMP-1 expression and c-Jun activation in a dose-dependent manner. We also observed that trans-Zeatin inhibits UVB-induced phosphorylation of ERK, JNK and p38 MAP kinases (MAPKs) dose-dependently. As expected, PD98059, an ERK inhibitor, SP600125, a JNK inhibitor and SB203580, a p38 MAPK inhibitor effectively inhibit UVB-induced phosphorylation of ERK, JNK and p38 MAPKs, respectively. Moreover, the inhibitory mechanism of trans-Zeatin was further demonstrated in MMP-1 secretion using MAPK-specific inhibitors. PD98059, SP600125 and SB203580 suppressed UVB-induced MMP-1 secretion, which is consistent with the above results. Collectively, our results suggest that trans-Zeatin inhibits UVB-induced MMP-1 expression, which may be
\end{abstract}

Correspondence to: Dr Zhigang Bi, Department of Dermatology, the First Affiliated Hospital of Nanjing Medical University, Nanjing 210029, Jiangsu Province, P.R. China

E-mail: eltonbi@21cn.com

Dr Yinsheng Wan, Department of Biology, Providence College, Providence, RI 02918, USA

E-mail: yswan@providence.edu

*Contributed equally

Key words: ultraviolet, matrix metalloproteinase-1, MAP kinase, trans-Zeatin, fibroblasts mediated by inhibition of ERK, JNK and p38 MAPKs signaling pathways in HSFs. Trans-Zeatin is a potential agent for the management of skin photoaging.

\section{Introduction}

Skin is the most susceptible organ to damage by solar ultraviolet (UV) radiation, as it is directly exposed to UV light (1). The solar UV light reaching earth comprises of a UVA wavelength (320-400 nm) and UVB wavelength (280$320 \mathrm{~nm}$ ). These two UV wavelengths are distinguished by their penetration properties $(2,3)$. UVB radiation reaches the upper part of the epidermis, a lesser extent of it, while UVA radiation penetrates more deeply into human skin $(3,4)$. However, although showing obvious differences, both UVA and UVB irradiation are responsible for the numerous adverse biological effects on human skin, including sunburn, immunosuppression, as well as long-term consequences such as skin cancer and premature skin aging or photoaging $(5,6)$. Amongst these various deleterious effects of UV irradiation, skin cancer and photoaging cause great social concern (6).

Clinically, photoaged skin is characterized by coarse wrinkles, thickening, rough texture and mottled pigmentation (7). Histological and ultrastructural studies revealed that the most prominent modifications in photoaged skin are in the dermis $(7,8)$. These UV-induced changes comprise an excessive deposition of degenerative elastotic material, decrease in collagen fibrils and alteration of the ratios between different types of collagen $(3,9,10)$. Since elastin and collagen fibrils are responsible for the strength and resiliency of the skin, their disarrangement in photoaging causes the skin to age (7).

It is proposed that UV irradiation leads to direct or indirect DNA damage and the formation of radical oxygen species (ROS) $(11,12)$, which causes the subsequent activation of complex signaling pathways, followed by matrix metalloproteinases (MMPs) induction in skin cells $(13,14)$. Furthermore, UV irradiation also activates growth factor receptors, which induce the activation of protein kinase cascades such as the MAP kinase (MAPK) signal transduction pathways in the cells (14-18). The MAPK signaling pathways regulate a variety of cellular functions, including the expression of MMPs and the inhibition of collagen production, which interferes with the metabolism of extracellular matrix (ECM) and causes the characteristic changes of photoaging in histo- 
pathology and clinical manifestation (11,19-21). MMPs are a family of zinc-dependent endoproteinases which are capable of degrading all components of ECM in skin photoaging. It is suggested that inhibition of induction of MMPs reduces UVinduced photoaging by preventing it from collagen degradation (22). Therefore, the agents which decrease the level of MMPs' production are the main focus of recent research.

Zeatin, purified from Zea mays, is a cytokinin in the group of plant growth factors (23). It has two forms, trans and cis. The activity of zeatin is attributed to its more stable trans form (24). Zeatin is one of the most widely studied cytokinins with growth modulatory and anti-senescence effects in plants (25). A previous study showed that Zeatin possesses a variety of biological activities, such as anti-apoptotic efficacy, antioxidative activity, youth preserving and anti-aging effects on the human system (25). However, there are no studies which report the effects of Zeatin on skin photoaging.

Given the potential biological activities of Zeatin, we undertook this study to investigate the effect of trans-Zeatin on UVB-induced matrix metalloproteinases-1 (MMP-1) expression in human skin fibroblasts (HSFs) and to further understand the mechanisms of its action. Our results demonstrate that treatment with trans-Zeatin inhibits UVBinduced MMP-1 expression, which is mediated by inhibition of MAPKs cell signaling in HSFs.

\section{Materials and methods}

Pharmacological agents. Anti-human MMP-1 antibody was purchased from Oncogene (Boston, MA), human phosphoextracellular signal-regulated kinase $1 / 2$ (ERK1/2), phosphoc-Jun N-terminal kinase (JNK), phospho-p38 mitogenactivated protein kinase (p38 MAPKs), $\beta$-actin and horseradish peroxidase-conjugated goat anti-mouse secondary antibodies were obtained from Santa Cruz Biotechnology (Santa Cruz, CA). Phospho-c-jun antibody was purchased from Cell signaling Technology (Beverly, MA). MAPK inhibitors (PD98059, SP600125, SB203580) were purchased from Sigma (Saint Louis, MO).

Cell culture. HSFs (derived from newborn skin) were acquired from the Chinese Academy of Medical Science. The cells were then cultured in Dulbecco's modified Eagle's medium (DMEM) (Hyclone, USA), supplemented with $10 \%$ fetal calf serum (FCS) (Invitrogen, AU), $100 \mathrm{U} / \mathrm{ml}$ penicillin and $100 \mu \mathrm{g} / \mathrm{ml}$ streptomycin. HSFs were cultivated in $75 \mathrm{~cm}^{2}$ culture flasks, in an incubator at $37^{\circ} \mathrm{C}$ humidified atmosphere containing $5 \%$ carbon dioxide. When they reached $80-90 \%$ confluency, the cells were subcultivated to $60 \mathrm{~mm}$ culture dishes.

UVB irradiation. As previously described $(15-18,26)$, four F36T12 ERE-VHO UV tubes were used in this study as the UV source. A Kodacel TA401/407 filter was mounted $4 \mathrm{~cm}$ in front of the tubes to block UVC (wavelengths below $290 \mathrm{~nm}$ ). The irradiation intensity was monitored by UVR radiometer equipped with a UVB sensor (Bioblock Scientific, Belgium). Subconfluent HSFs were cultured in DMEM containing $0.1 \%$ FCS for $24 \mathrm{~h}$, and subsequently incubated in DMEM with various concentrations of trans-Zeatin (Sigma, China) for $24 \mathrm{~h}$. HSFs were then washed twice with fresh phosphate buffer saline (PBS) and exposed to UVB irradiation $\left(30 \mathrm{~mJ} / \mathrm{cm}^{2}\right)$ in a thin layer of PBS. After irradiation, the cells were incubated in DMEM for the indicated time.

Cell viability assay. Briefly, HSFs were seeded in a 96-well plate. After various concentrations of trans-Zeatin or UVB treatment, cells were cultured in $100 \mu 110 \%$ DMEM in each well for the indicated time. MTT solution $(10 \mu 1)$ at a concentration of $5 \mathrm{mg} / \mathrm{ml}$ was added to each well, and the cells were then incubated at $37^{\circ} \mathrm{C}$ for $4 \mathrm{~h}$. The medium was removed, and $200 \mu 1$ of Dimethyl-sulfoxide (DMSO, Sigma, St. Louis, MO) was added into each well to dissolve formazan crystals by pipetting up and down several times. The absorbance was measured spectrophotometrically on an ELISA plate reader at a wavelength of $570 \mathrm{~nm}$.

Enzyme-linked immunosorbent assay. Amount of MMP-1 in the culture supernatant, including the active form, was quantified by an enzyme-linked immunosorbent assay kit (BPB Biomedicals Inc, USA). Samples were measured in triplicates according to the manufacturer's instructions.

Western blot analysis. HSFs were homogenized in lysis buffer (20 mM Tris [pH 8.0], $150 \mathrm{mM} \mathrm{NaCl}, 1 \% \mathrm{NP}-40,1 \%$ deoxycholate, $1 \mathrm{mM}$ EDTA and phenylmethylsulfonyl fluoride each, $40 \mathrm{mM}$ glycerophosphate, $125 \mu \mathrm{M} \mathrm{Na}_{3} \mathrm{VO}_{4}, 50 \mathrm{mM} \mathrm{NaF}$, $2 \mu \mathrm{g} / \mathrm{ml}$ of leupeptin, aprotinin, pepstatin each, and $1 \mathrm{mM}$ dithiothreitol). Equal amount of proteins or equal volume of culture supernatants were electrophoresed on a $10 \%$ sodium dodecyl sulfate-polyacrylamide gel (SDS-PAGE). Proteins were transferred onto polyvinylidene fluoride (PVDF) membranes. After blocking with $3 \%$ bovine serum albumin (BSA) in TBST buffer (20 mM Tris- $\mathrm{HCl}$ [pH 7.6], $137 \mathrm{mM}$ $\mathrm{NaCl}, 0.05 \%$ Tween-20), the membranes were incubated with indicated antibodies overnight at $4^{\circ} \mathrm{C}$. Being washed three times with TBST, the blots were incubated with horseradish peroxidase-conjugated goat anti-mouse secondary antibody for $1 \mathrm{~h}$ at room temperature. Blotted proteins were visualized by enhanced chemiluminescence (Pierce, USA). The density of the bands was obtained using Quantity-One software (Bio-Rad, USA).

Statistical analysis. Data were statistically analyzed using the SPSS 11.0 Software. Results were expressed as means \pm SD. Statistical analysis was determined by the Student's t-test. $\mathrm{P}<0.05$ was considered statistically significant.

\section{Results}

The effect of trans-Zeatin on cell viability in human skin fibroblasts. First, we tested whether trans-Zeatin affects cell viability in human skin fibroblasts using MTT assay. As shown in Fig. 1A, at a low concentration $(80 \mu \mathrm{M})$, trans-Zeatin did not affect cell viability. Trans-Zeatin at $150 \mu \mathrm{M}$ showed almost $95 \%$ cell viability. While the concentration was over $300 \mu \mathrm{M}$, trans-Zeatin had a cytotoxic effect on HSFs. We next examined the effect of trans-Zeatin on cell viability in UVB-exposed HSFs. The results showed that UVB irradiation $\left(30 \mathrm{~mJ} / \mathrm{cm}^{2}\right)$ showed slight cytotoxicity. Trans-Zeatin $(20-80 \mu \mathrm{M})$ enhanced cell viability in HSFs irradiated with UVB (Fig. 1B). In the 
A

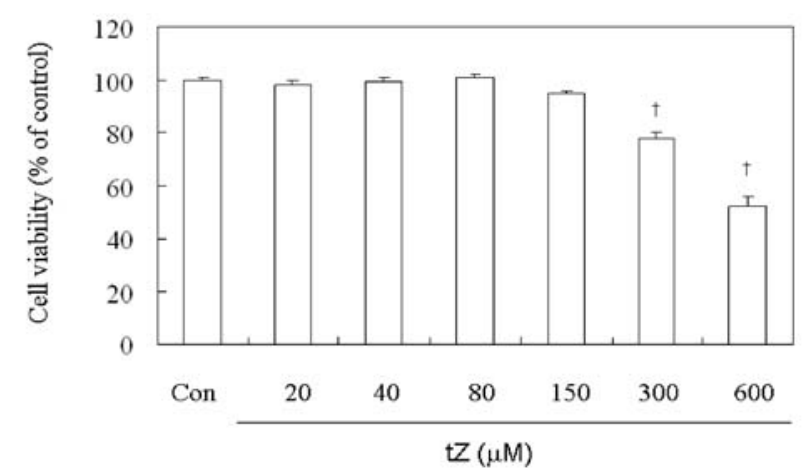

B

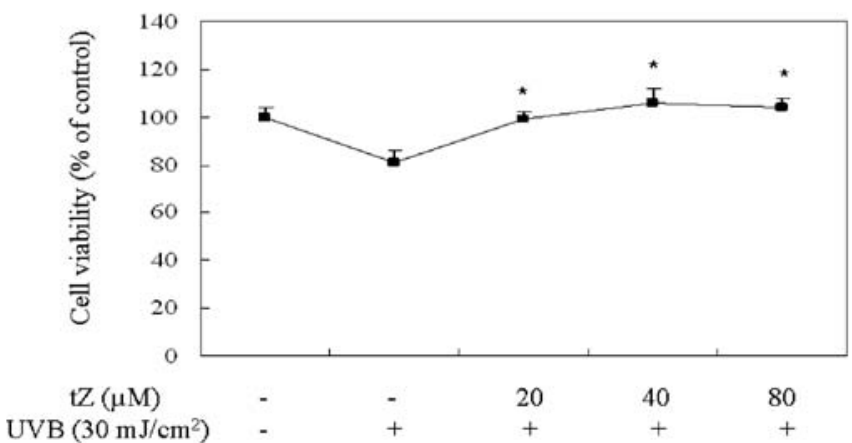

Figure 1. Effect of trans-Zeatin on cell viability in human skin fibroblasts. (A) Fibroblasts were treated with either presence or absence of trans-Zeatin (tZ) in medium for $24 \mathrm{~h}$. (B) The cells were pretreated with indicated concentrations of trans-Zeatin for $24 \mathrm{~h}$, followed by irradiation with UVB $\left(30 \mathrm{~mJ} / \mathrm{cm}^{2}\right)$, and the cells were then further cultured for $24 \mathrm{~h}$. The cytotoxicity was measured using MTT assay. The absorbance was read on a microplate reader at $570 \mathrm{~nm}$. Results are given as mean $\pm \mathrm{SD}$ of three independent experiments performed in triplicates. ${ }^{+} \mathrm{P}<0.05$ compared with the control group, ${ }^{*} \mathrm{P}<0.05$ compared with the only UVB-irradiated group.

following experiments, we used $30 \mathrm{~mJ} / \mathrm{cm}^{2} \mathrm{UVB}$ irradiation and 20-80 $\mu \mathrm{M}$ of trans-Zeatin.

Effect of trans-Zeatin on UVB-induced MMP-1 expression. It is well known that UV irradiation damages human skin cells and causes photoaging. UVA and UVB irradiation of dermal fibroblasts in vitro or human skin in vivo induces MMP-1 expression, which is implicated in the degradation of human skin matrix proteins such as collagen and other components of ECM $(20,22)$. In this study, to investigate whether transZeatin affects the expression of MMP-1 in UVB-irradiated HSFs, cultured fibroblasts were pretreated with various concentrations of trans-Zeatin followed by UVB irradiation. MMP-1 protein levels were determined by ELISA and Western blotting respectively. As expected, the results showed that UVB irradiation significantly enhanced MMP-1 expression. ELISA results showed that pretreatment with trans-Zeatin markedly inhibited UVB-induced MMP-1 expression in a dosedependent manner compared to only UVB-irradiated group (Fig. 2A). Similar results were observed by Western blotting (Fig. 2B).

Inhibitory effect of trans-Zeatin on UVB-induced ERK, JNK, p38 MAPK and c-Jun phosphorylation. Significant progress has been made towards understanding the molecular mechanisms underlying UV-induced MMPs expression. It was found that
A

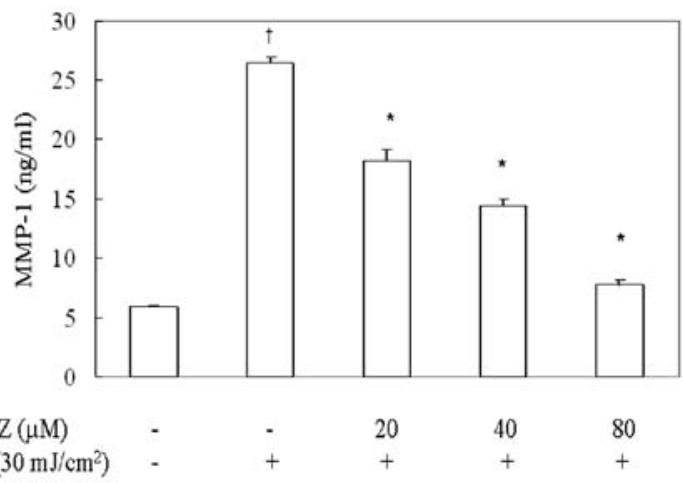

B

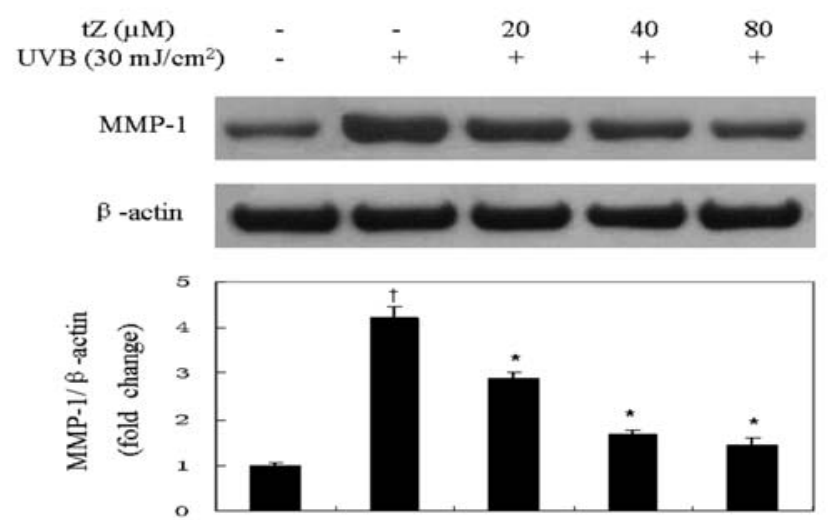

Figure 2. Effect of trans-Zeatin on UVB-induced MMP-1 expression. Human skin fibroblasts were pretreated with indicated concentrations of trans-Zeatin (tZ) for $24 \mathrm{~h}$, followed by irradiation with UVB $\left(30 \mathrm{~mJ} / \mathrm{cm}^{2}\right)$, and then incubated for $24 \mathrm{~h}$. (A) The culture supernatants were harvested and MMP-1 protein in the supernatants was determined with a sandwich immunoassay kit. (B) The cells were harvested, and MMP-1 expression was determined by Western blotting. Results are given as mean $\pm \mathrm{SD}$ of three independent experiments performed in triplicates. ${ }^{+} \mathrm{P}<0.05$ compared with the control, ${ }^{*} \mathrm{P}<0.05$ compared with the only UVB-irradiated group.

UV radiation activates growth factor receptors, which induces the activation of the MAPKs cascade. This activation is then followed by an increased expression of c-Jun and -Fos, which form the activator protein-1 (AP-1) complex with transcriptions of several MMPs, including MMP-1 (27-29). To determine the possible mechanisms involved in trans-Zeatin's effect on UVB-induced MMP-1 expression, we further studied the effect of trans-Zeatin on UVB-induced activation of ERK1/2, JNK and p38 MAPKs in HSFs. PD98059, SP600125 and SB203580 were employed as positive controls respectively. As shown in Fig. 3A, B and C, trans-Zeatin inhibited UVBinduced ERK1/2, JNK and p38 MAPKs phosphorylation dosedependently, while having weak inhibitory effect on phosphorylation of ERK1/2 (20 and $40 \mu \mathrm{M})$ and p38 MAPKs $(20 \mu \mathrm{M})$. As MAPKs pathways influence AP-1 transactivation by increasing the level of AP-1 components or by altering the phosphorylation of their subunits, such as c-Jun $(27,30)$, subsequently, we also investigated the effect of trans-Zeatin on the activation of c-Jun. The results showed that phosphorylation of c-Jun induced by UVB irradiation was significantly attenuated by trans-Zeatin (Fig. 3D).

Effect of MAP kinase inhibitors on UVB-induced MMP-1 secretion. The results above suggests that trans-Zeatin inhibits 
A
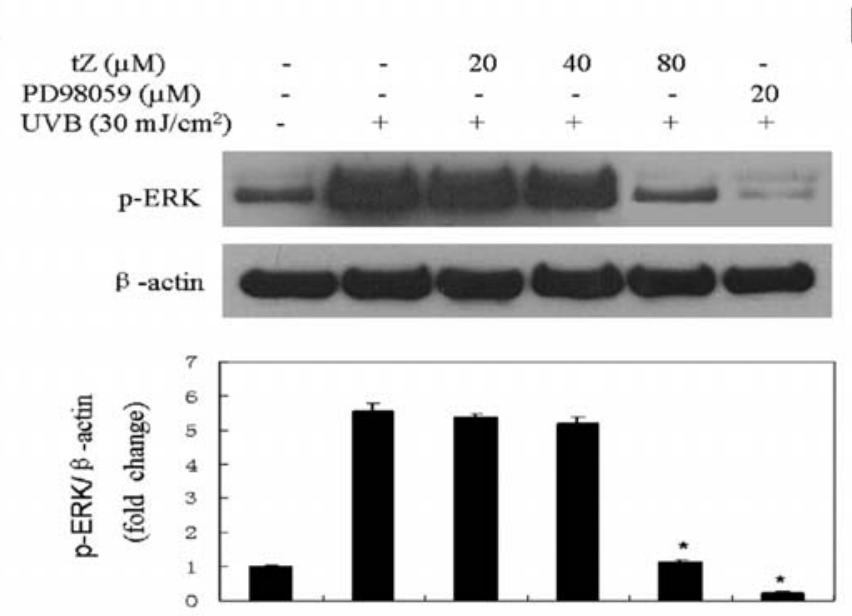

C
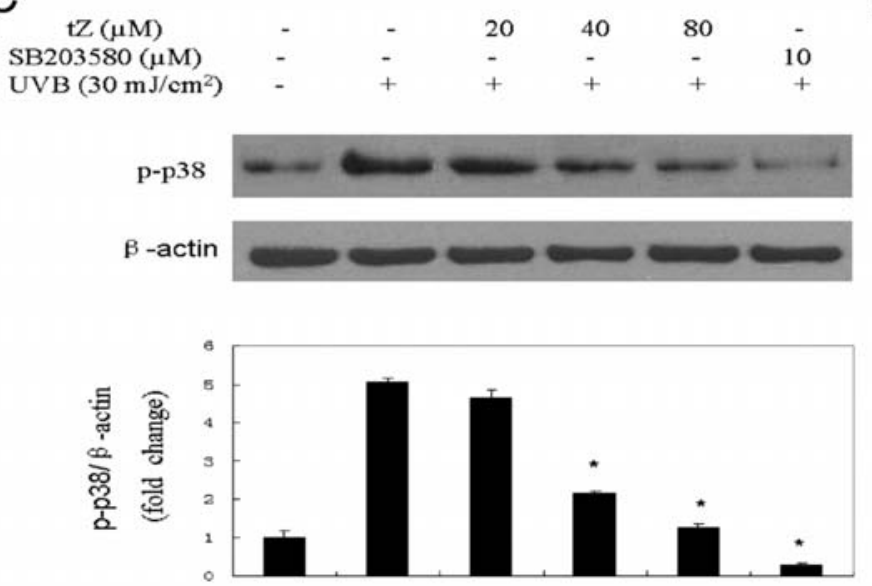
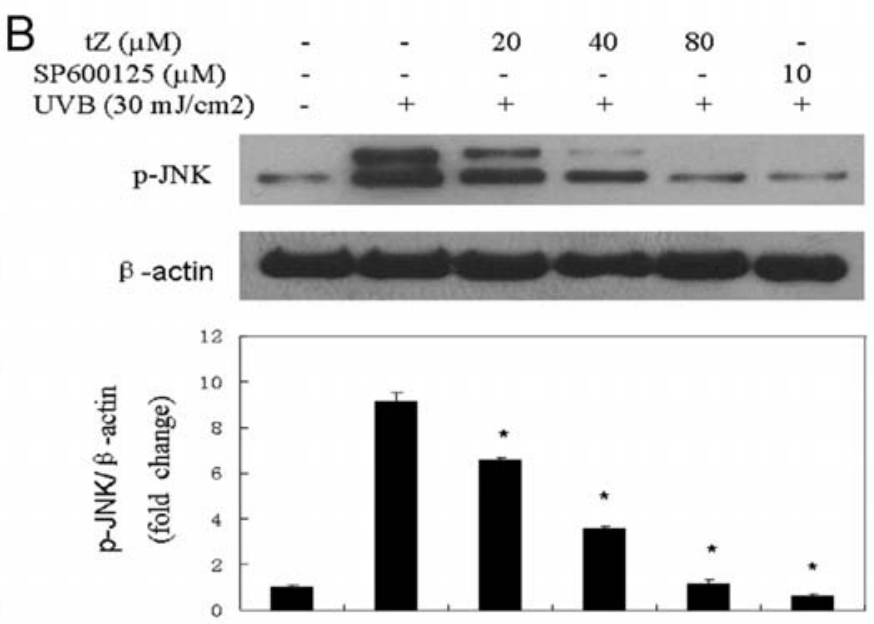

D $\begin{array}{cccccr}\mathrm{tZ}(\mu \mathrm{M}) & - & - & 20 & 40 & 80 \\ \mathrm{UVB}\left(30 \mathrm{~mJ} / \mathrm{cm}^{2}\right) & - & + & + & + & +\end{array}$

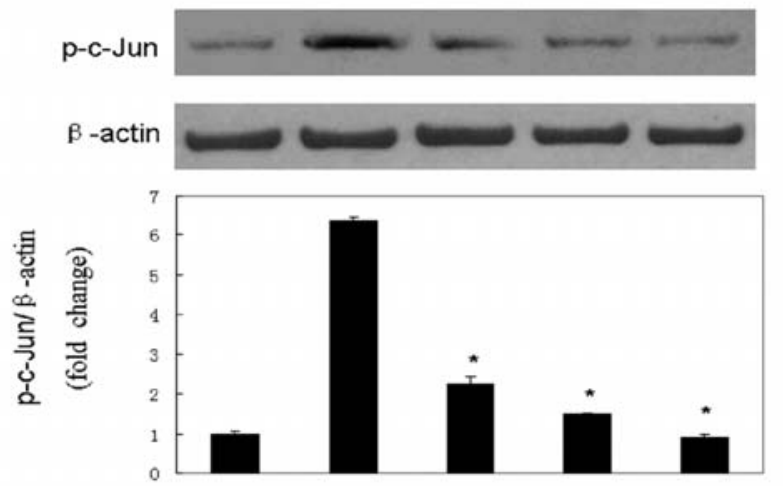

Figure 3. Effect of trans-Zeatin on UVB-induced ERK, JNK, p38MAPKs and c-Jun phosphorylation. Human skin fibroblasts were pretreated with indicated concentrations of trans-Zeatin for $24 \mathrm{~h}$ or MAPK inhibitors for $2 \mathrm{~h}$, followed by UVB $\left(30 \mathrm{~mJ} / \mathrm{cm}^{2}\right)$ irradiation, the cells were then incubated for $30 \mathrm{~min}$ or $1 \mathrm{~h}$ respectively, the phosphorylation of ERK (A), JNK (B), p38 (C) MAPKs and c-Jun (D) was determined by Western blotting. Results are given as mean \pm SD of three independent experiments. ${ }^{*} \mathrm{P}<0.05$ compared with the only UVB-irradiated group.

MMP-1 expression via inhibition of ERK1/2, JNK and p38 MAPKs dependent signaling pathways. To confirm the results, we pretreated HSFs with specific kinase inhibitors of each MAPK (20 $\mu \mathrm{M}$ PD098059, ERK inhibitor; $10 \mu \mathrm{M}$ SP600125, JNK inhibitor; $10 \mu \mathrm{M} \mathrm{SB} 203580$, p38 kinase inhibitor). After UVB irradiation, secreted MMP-1 in the supernatants were harvested and determined by Western blotting. As shown in Fig. 4, UVB-induced MMP-1 secretion was inhibited by all three MAPKs inhibitors, while SP600125 showed the strongest inhibitory effect. These results suggest that UVB-induced MMP-1 secretion in HSFs is mediated by the activation of the ERK1/2, JNK and p38 MAPKs signaling pathways. The findings further suggest that inhibition of MMP-1 expression by trans-Zeatin is partially mediated via the suppression of ERK1/2, JNK and p38 MAPKs signaling pathways in UVBirradiated HSFs.

\section{Discussion}

In recent years, more changes in lifestyle caused a significant increase in the number of people overexposed to UV irradiation, leading to a surge in the incidence of skin photoaging (6). As these trends are likely to continue in the

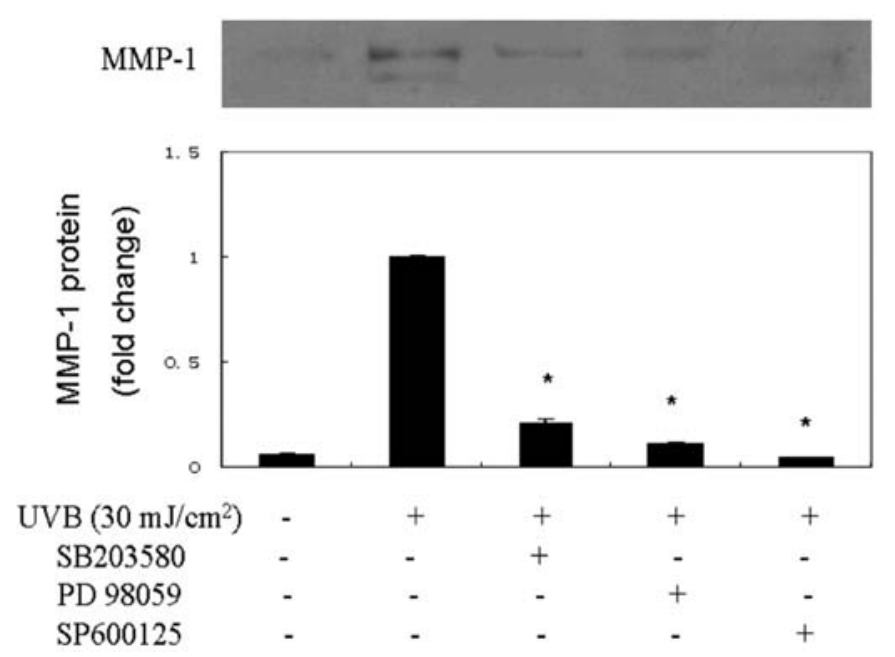

Figure 4. Effects MAPK inhibitors on UVB-induced MMP-1 secretion. Human skin fibroblasts were treated with ERK inhibitor PD98059 (20 $\mu \mathrm{M})$, JNK inhibitor SP600125 $(10 \mu \mathrm{M})$, and p38 MAPK inhibitor SB203580 $(10 \mu \mathrm{M})$ respectively, then irradiated with UVB $\left(30 \mathrm{~mJ} / \mathrm{cm}^{2}\right)$. Secreted MMP-1 $48 \mathrm{~h}$ after irradiation was determined by Western blotting. Results are given as mean \pm SD of three independent experiments. ${ }^{*} \mathrm{P}<0.05$ compared to the only UVB-irradiated group. 
foreseeable future, development of novel strategies to alleviate UV-induced photoaging are highly desirable (6). Botanical antioxidants and natural compounds have gained considerable attention as photoaging preventive agents for human use. Zeatin, purified from Zea mays, is a member of the cytokinin group of plant growth factors (23). There is a large amount of information available regarding the activity of Zeatin in bacteria, fungi and higher plants (25). However, there is relatively little attention to the biological effects of Zeatin on the human system so far. In the present study, we investigated the inhibitory effect and action mechanism of trans-Zeatin on UVB-induced MMP-1 expression in HSFs.

UV irradiation is known to induce expressions of MMP$1,-3$, and -9 in human skin in vivo and cultured human skin cells in vitro (31-33). UV-induced MMP-1 expression induces the cleavage of collagen fibers. Once collagen is cleaved by MMP-1, collagen degradation is further promoted by MMP-3 and -9 (34). MMP-1, termed fibroblast-type or interstitial collagenase, is secreted by keratinocytes, fibroblasts, and macrophages. MMP-1 degrades collagen types I, II, and III, and is thought to play a pivotal role in the process of photoaging. These properties make MMP-1 an attractive target for the pharmacological development of anti-photoaging agents. Therefore, in this study, we examined the effect of trans-Zeatin on MMP-1 expression. We found that trans-Zeatin markedly suppresses UVB-induced MMP-1 expression in a dosedependent manner in HSFs (Fig. 2). The results suggest that trans-Zeatin prevents UVB-induced collagen damage by inhibiting UVB-induced MMP-1 expression.

In vivo, transcription of MMP-1 is regulated by AP- 1 . The downstream effectors of MAPKs include several transcription factors, such as c-Fos and -Jun. For the formation of AP-1 complex, Jun proteins (Jun B, c-Jun, and Jun D) form homodimers or heterodimers with Fos proteins (Fos B, c-Fos, Fra-1, and -2) (35). In human skin, the transcriptional activity of AP-1 is also limited by the degree of phosphorylation of c-Jun $(27,30)$. Our data show that trans-Zeatin efficiently inhibits UVB-induced phosphorylation of c-Jun (Fig. 3D). Therefore, it is possible that trans-Zeatin inhibits UVBinduced MMP-1 expression via suppressing UVB-induced c-Jun activation. MAPK signaling pathways play an important role in regulating a variety of cellular functions, including MMPs expression $(14,27,29)$. MAPKs contain three general classes: ERK, JNK, and p38 MAPKs, mediating AP-1 activation. Activation of MAPKs occurs through the phosphorylation of specific threonines and tyrosines, and the components of AP-1 are phosphorylated after the activated MAPKs translocate to the nucleus (36). In this study, we found that trans-Zeatin inhibits UVB-induced activation of ERK, JNK and p38 MAPKs (Fig. 3A, B, and C). Furthermore, PD98059, an ERK inhibitor, SP600125, a JNK inhibitor and SB203580, a p38 MAPK inhibitor effectively inhibit UVB-induced phosphorylation of ERK, JNK and p38 MAPKs respectively, suggesting that trans-Zeatin suppresses MMP-1 expression via the ERK, JNK and p38 MAPKs signaling pathways. The inhibitory mechanism of trans-Zeatin was further demonstrated in the MMP-1 secretion using MAPK specific inhibitors. As shown in Fig. 4, PD98059, SP600125 and SB203580 suppress UVB-induced MMP-1 secretion, which is consistent with the results shown in
Fig. 3A, B and C. These data further suggest that transZeatin inhibits UVB-induced MMP-1 expression, which may be through the suppression of ERK, JNK and p38 MAPKs signaling.

It was shown that different natural compounds or botanical antioxidants with similar perspectives have different action mechanisms. For example, it was observed that epigallocatechin-3-gallate (EGCG), a polyphenolic compound inhibits UV-induced MMP-1 expression via inhibiting activation of c-Jun, -fos and p53, downstream proteins of JNK, p38 MAPK and ERK1/2 (29). In addition, a previous study found that obovatol inhibits UVB-induced MMP-3 expression through inhibition of the activation of ERK, p38 MAPK, but not JNK signaling (37). Our results support the notion that trans-Zeatin inhibits UVB-induced MMP-1 expression via inhibiting ERK, JNK and p38 pathways.

In summary, our study demonstrates that trans-Zeatin inhibits UVB-induced MMP-1 expression, which is mediated by inhibition of ERK, JNK and p38 MAPKs signaling pathways in HSFs. Our data suggest that trans-Zeatin is a potential agent for the management of skin photoaging.

\section{Acknowledgements}

This research was supported in part by grants from the National Natural Science Foundation of China (30271195 and 30671894) and by a grant from NIH (P20 RR016457 from INBRE Program of the National Center for Research Resources).

\section{References}

1. Soo Lee Y, Jin DQ, Beak SM, Lee ES and Kim JA: Inhibition of ultraviolet-A-modulated signaling pathways by asiatic acid and ursolic acid in $\mathrm{HaCaT}$ human keratinocytes. Eur J Pharmacol 476: 173-178, 2003.

2. Bruls WA, Slaper H, van der Leun JC and Berrens L: Transmission of human epidermis and stratum corneum as a function of thickness in the ultraviolet and visible wavelengths. Photochem Photobiol 40: 485-494, 1984.

3. Fagot D, Asselineau D and Bernerd F: Matrix metalloproteinase-1 production observed after solar-simulated radiation exposure is assumed by dermal fibroblasts but involves a paracrine activation through epidermal keratinocytes. Photochem Photobiol 79: 499-505, 2004

4. Xia J, Song X, Bi Z, Chu W and Wan Y: UV-induced NF-kappaB activation and expression of IL-6 is attenuated by (-)-epigallocatechin-3-gallate in cultured human keratinocytes in vitro. Int $\mathrm{J}$ Mol Med 16: 943-950, 2005.

5. Young AR: Cumulative effects of ultraviolet radiation on the skin: cancer and photoaging. Semin Dermatol 9: 25-31, 1990.

6. Afaq $\mathrm{F}$ and Mukhtar $\mathrm{H}$ : Botanical antioxidants in the prevention of photocarcinogenesis and photoaging. Exp Dermatol 15: 678-684, 2006.

7. Fisher GJ, Datta S, Wang Z, et al: c-Jun-dependent inhibition of cutaneous procollagen transcription following ultraviolet irradiation is reversed by all-trans retinoic acid. J Clin Invest 106: 663-670, 2000.

8. Mitchell RE: Chronic solar dermatosis: a light and electron microscopic study of the dermis. J Invest Dermatol 48: 203-220, 1967.

9. Brinckmann J, Acil Y, Wolff $\mathrm{HH}$ and Muller PK: Collagen synthesis in (sun-) aged human skin and in fibroblasts derived from sun-exposed and sun-protected body sites. J Photochem Photobiol B 27: 33-38, 1995.

10. Talwar HS, Griffiths CE, Fisher GJ, Hamilton TA and Voorhees JJ: Reduced type I and type III procollagens in photodamaged adult human skin. J Invest Dermatol 105: 285-290, 1995.

11. Jiang Q, Zhou C, Healey S, et al: UV radiation down-regulates Dsg-2 via Rac/NADPH oxidase-mediated generation of ROS in human lens epithelial cells. Int J Mol Med 18: 381-387, 2006. 
12. Kalbina I and Strid A: The role of NADPH oxidase and MAP kinase phosphatase in UV-B-dependent gene expression in Arabidopsis. Plant Cell Environ 29: 1783-1793, 2006.

13. Brenneisen $\mathrm{P}$, Wenk J, Klotz LO, et al: Central role of Ferrous/Ferric iron in the ultraviolet B irradiation-mediated signaling pathway leading to increased interstitial collagenase (matrix-degrading metalloprotease (MMP)-1) and stromelysin-1 (MMP-3) mRNA levels in cultured human dermal fibroblasts. J Biol Chem 273: 5279-5287, 1998.

14. Fisher GJ, Talwar HS, Lin J and Voorhees JJ: Molecular mechanisms of photoaging in human skin in vivo and their prevention by all-trans retinoic acid. Photochem Photobiol 69: 154-157, 1999

15. Cao C, Lu S, Kivlin R, et al: AMP-activated protein kinase contributes to UV- and $\mathrm{H}_{2} \mathrm{O}_{2}$-induced apoptosis in human skin keratinocytes. J Biol Chem 283: 28897-28908, 2008.

16. Cao C, Lu S, Kivlin R, et al: SIRT1 confers protection against UVB- and $\mathrm{H}(2) \mathrm{O}(2)$-induced cell death via modulation of p53 and JNK in cultured skin keratinocytes. J Cell Mol Med (In press).

17. Cao C, Wan S, Jiang Q, et al: All-trans retinoic acid attenuates ultraviolet radiation-induced down-regulation of aquaporin-3 and water permeability in human keratinocytes. J Cell Physiol 215: 506-516, 2008

18. Chen W, Kang J, Xia J, et al: p53-related apoptosis resistance and tumor suppression activity in UVB-induced premature senescent human skin fibroblasts. Int J Mol Med 21: 645-653, 2008.

19. Yan SX, Hong XY, Hu Y and Liao KH: Tempol, one of nitroxides, is a novel ultraviolet-A1 radiation protector for human dermal fibroblasts. J Dermatol Sci 37: 137-143, 2005.

20. Wlaschek M, Briviba K, Stricklin GP, Sies H and Scharffetter-Kochanek K: Singlet oxygen may mediate the ultraviolet A-induced synthesis of interstitial collagenase. J Invest Dermatol 104: 194-198, 1995.

21. Kang J, Chen W, Xia J, et al: Extracellular matrix secreted by senescent fibroblasts induced by UVB promotes cell proliferation in $\mathrm{HaCaT}$ cells through PI3K/AKT and ERK signaling pathways. Int J Mol Med 21: 777-784, 2008.

22. Fisher GJ, Datta SC, Talwar HS, et al: Molecular basis of suninduced premature skin ageing and retinoid antagonism. Nature 379: 335-339, 1996.

23. Koenig RL, Morris RO and Polacco JC: tRNA is the source of low-level trans-zeatin production in Methylobacterium spp. J Bacteriol 184: 1832-1842, 2002.

24. Kulaeva ON, Karavaiko NN, Selivankina S, Zemlyachenko Ya V and Shipilova SV: Receptor of trans-zeatin involved in transcription activation by cytokinin. FEBS Lett 366: 26-28, 1995.
25. Rattan SI and Sodagam L: Gerontomodulatory and youthpreserving effects of zeatin on human skin fibroblasts undergoing aging in vitro. Rejuvenation Res 8: 46-57, 2005.

26. Fisher GJ, Kang S, Varani J, et al: Mechanisms of photoaging and chronological skin aging. Arch Dermatol 138: 1462-1470, 2002.

27. Kim HH, Shin CM, Park CH, et al: Eicosapentaenoic acid inhibits UV-induced MMP-1 expression in human dermal fibroblasts. J Lipid Res 46: 1712-1720, 2005.

28. Fisher GJ and Voorhees JJ: Molecular mechanisms of photoaging and its prevention by retinoic acid: ultraviolet irradiation induces MAP kinase signal transduction cascades that induce Ap-1regulated matrix metalloproteinases that degrade human skin in vivo. J Investig Dermatol Symp Proc 3: 61-68, 1998.

29. Bae JY, Choi JS, Choi YJ, et al: (-)Epigallocatechin gallate hampers collagen destruction and collagenase activation in ultraviolet-B-irradiated human dermal fibroblasts: involvement of mitogen-activated protein kinase. Food Chem Toxicol 46: 1298-1307, 2008.

30. Bode AM and Dong Z: Signal transduction pathways: targets for chemoprevention of skin cancer. Lancet Oncol 1: 181-188, 2000.

31. Petersen MJ, Hansen C and Craig S: Ultraviolet A irradiation stimulates collagenase production in cultured human fibroblasts. J Invest Dermatol 99: 440-444, 1992.

32. Fisher GJ, Wang ZQ, Datta SC, Varani J, Kang S and Voorhees JJ: Pathophysiology of premature skin aging induced by ultraviolet light. N Engl J Med 337: 1419-1428, 1997.

33. Oh JH, Kim A, Park JM, Kim SH and Chung AS: Ultraviolet Binduced matrix metalloproteinase- 1 and -3 secretions are mediated via PTEN/Akt pathway in human dermal fibroblasts. J Cell Physiol 209: 775-785, 2006.

34. Kim HH, Cho S, Lee S, et al: Photoprotective and anti-skinaging effects of eicosapentaenoic acid in human skin in vivo. J Lipid Res 47: 921-930, 2006.

35. Huang C, Schmid PC, Ma WY, Schmid HH and Dong Z: Phosphatidylinositol-3 kinase is necessary for 12-Otetradecanoylphorbol-13-acetate-induced cell transformation and activated protein 1 activation. J Biol Chem 272: 4187-4194, 1997.

36. Lee J, Jung E, Lee J, et al: Emodin inhibits TNF alpha-induced MMP-1 expression through suppression of activator protein-1 (AP-1). Life Sci 79: 2480-2485, 2006.

37. Choi MS, Yoo MS, Son DJ, et al: Increase of collagen synthesis by obovatol through stimulation of the TGF-beta signaling and inhibition of matrix metalloproteinase in UVB-irradiated human fibroblast. J Dermatol Sci 46: 127-137, 2007. 